\title{
Distribution of benthic communities affected by human activities in the west coast of Alexandria, Mediterranean Sea, Egypt
}

\author{
Mohamed M. El-Komi \\ National Institute of Oceanography and Fisheries, Alexandria, Egypt \\ Komimohamed50@yahoo.com
}

\begin{abstract}
In the framework of marine ecosystem evaluation, this study was carried out where sediment microorganisms were collected bimonthly from 16 sites over one year in the western coast of Alexandria. This area comprises different human activities (urban sewage, agriculture irrigation, industrial wastes, petroleum spill, tourist stress, fishing and commercial navigation). Macrobenthos communities are well indicators of water and sediment quality. Bottom invertebrate's diversity, comparison of benthos communities at different sampling sites and the biomass were determined as well as the numerical abundance of benthos in comparison to the different environmental parameters. Discharging of chemical pollutants in coastal waters leads to significant degradation in the water quality that altered the macro invertebrates' diversity indices. The most western sites are less stressed area indicating low diversity rather than the stressed area close to the main sources of pollutants discharge that has relatively high pollution and generally with good diversity. The benthic communities are dominated by few species of pollution tolerance which are used as indicators of polluted sediments such as free-living nematodes and polychaetes. The regional distribution of benthic invertebrate's communities varied at different sites and over the period of collections that total abundance ranged from 959 to 8444 ind. $/ \mathrm{m}^{2}$ with an annual average of 3072 ind. $/ \mathrm{m}^{2}$ and seven benthos groups can be sequenced as follows: Nematodes $46 \%>$ polychaetes $22 \%>$ amphipods $13.8 \%>$ cirripedes $4.8 \%>$ oligochaetes $4 \%>$ gastropods $3.0 \%>$ bivalves $1.5 \%$. On the other hand, biomass of benthic structure has an annual average of $155 \mathrm{~g} / \mathrm{m}^{2}$, where six predomination benthic groups can be ranked in the following sequence: Cirripedes 33.5\% > Algae 30.6\% > Gastropods $12.7 \%>$ Bivalves $9.1 \%>$ Sea grass $7.0 \%>$ Polychaetes $1.7 \%$.

At the different sites, the general structure of species number of benthos groups indicated that Polychaeta was the most common group comprising 27 species of 80 the total number of taxa. The values of species diversity $\left(\mathrm{H}^{\prime}\right)$ had relatively moderate diversity $(1.3-2.02)$ at most stations and in general the decline in diversity is primarily attributed to the uneven distribution of individuals among the species. On the other hand, the species diversity was only 1.6 at Site 2 though it was the richest that may be attributed to the number of species. Values of the evenness index indicated that sites $1,8,9,10$ were highly diverse in benthic communities. The Swartz's dominance index value indicated less stress with high diversity and number of species. The area located close to the outfall of the two main drains are characterized by having distinct eutrophication level leading to significant increase in the species richness and mean abundance of benthic communities of the tolerant species. Continuous monitoring and assessing of macrobenthic communities is needed to determine the short and long-term changes in the marine ecosystem that are essential for fisheries management along the coastal region.
\end{abstract}

Key words: macro benthos, impact of pollution, biodiversity, benthic communities, polychaetes, bivalves, crustaceans. 


\section{INTRODUCTION}

Coastal developmental evaluation at the western side of Alexandria, at the south eastern region of the Mediterranean Sea, has attracted little attention in particular to the effects of industrial, tourist activities and marine transport on the physical, chemical and biological parameters of water quality and fish population. Recently, due to increasing of human activities, the marine ecosystem is threatened by the direct effects of the public and private resort beaches, marine transport, along the Western and El Dehkela Harbors, fish vessels, irrigation and industrial discharges through El Amom and Nobaria drains. These areas received a large input of nutrients, sediment and organic matter from the different pollutant sources but limited primary production from benthic microalgae and water column phytoplankton. Some benthic fauna are adapted to response to pollution due to organic enrichment but many species are immigrating to avoid prolonged exposure. The presence of any species in any area is dependant on its tolerance to those environmental variables since considerable spatial and temporal variations occur within estuarine and coastal sedimentary areas. Benthic communities used as biological indicators for the impact of human activities (Rees et al., 2005; Borja and Muxika, 2005). The eutrophication and hypoxia/anoxia (Rumohr, 2005) are acute threats to Baltic biota, and the benthic communities due to sharp environmental gradients and the increasing anthropogenic influences. Organic matter in sediments is a source of food for benthic fauna (Shine, 2005) higher levels can cause reduction in biodiversity indices. The area located close to the outfall of the main drains are characterized by having distinct eutrophication leading to significant increase in the species richness and mean abundance of benthic communities of the tolerant species.

In general, the benthos community is becoming an important item in studying of aquatic food webs which represent a good indicator for the impact of pollution on the aquatic ecosystem. The seas have been considered a dependable provider of protein - rich food and can be able to provide all the food for humanity needs. The occurrence of the bottom living organisms of both the infauna and epifauna providing well information about the feeding habits of other members of living organisms as a food source for fishes in particular, and to extend our knowledge of trophic interaction in the sea. The effect of hydrobiological disturbances is either biotic or abiotic factors on the composition, abundance, and distribution of species within benthic communities where the infauna and epifauna have wider range of tolerance than the mobile fauna. Thus, the mobility of benthic communities may be an adaptation to unstable substrata and frequent hydrologic disturbance. Abiotic disturbance related to the application of a damaging physical factor to the habitat of a population community or ecosystem (Lake, 2000). This includes any biotic factor which causes physiological stress to organisms but does not necessarily reduce the number of individuals (Thomson et al., 2002). The responses of benthos to disturbance of environmental conditions are clearly important and there is simple relationship between their relative tolerance and pollutants impacts. The confounding effects of pollution on the regional and spatial distribution of macro benthos as reported in numerous studies, can predict with confidence the relative impacts of the abiotic conditions through field surveys to estimate various biodiversity indices.

Nutrient discharged substances from sewage, industrial wastes and irrigation drainage (e.g. El Amom drain discharged 144-284 x $10^{6} \mathrm{~m}^{3}$ monthly) are not recycled, flow into the sea causing dense plankton bloom. This bloom sinks to deeper waters to be decomposed, requiring oxygen and may become anoxic. On the other hand, many 
marine organisms have the ability to concentrate certain levels of chemical pollutants inside their bodies. Particularly plankton organisms do so and those feeding on them. Therefore, these chemicals are concentrated once again to kill the secondary producers as birds and fish for human consumption. Oils may be toxic to aquatic life when ingested or absorbed through skin or gills, interfere with respiratory function of aquatic communities, degrading habitats and bathing beaches. Nevertheless, much oil is liquid, but gaseous hydrocarbons are volatile or easily degraded and mostly disappear from the aquatic system, while some may persist in the water column or in sediment. Heavy metals are stable and persistent as environmental contaminants, which cannot be degraded and tend to accumulate in the soils and sediments. High levels of metals in marine ecosystem affect on marine biota (seaweeds, benthic invertebrates) causing high mortality to fish, shrimps, and larvae of many kinds which feed on plankton and cause risk to human consumers of seafood.

The objective of macro-benthic distribution studies in this area is to determine bottom invertebrate's diversity, comparison of benthos communities at different sampling sites and the biomass as well as the numerical abundance of benthos in relation to the different environmental parameters. The main aim is to predict the effectiveness of different environmental impacts, regulations, and incentives in reducing the ecological risks and consequently of sedimentation to coastal regions. The main problem of sedimentation in coastal waters is the degradation of water quality, as it alters the habitat for fish and macro invertebrates, limits the ecosystem functions and services and reduces the aesthetic and economic values of coastal regions. The benthos data analyses of their species composition, density abundance, and biomass will be compared with the available parameters of the water quality and the sediment structure.

\section{MATERIAL AND METHODS}

\section{Area of Study}

This area is usually subjected to different kinds of human activities hence receiving large amount of fresh water coming from many drains mixed with the polluted water drained through different waste products such as industrial products and other pollutants discharged into the sea without any kind of treatment. Sixteen sites were selected to represent different habitats along the inshore region as shown in Fig. 1:

\begin{tabular}{|c|l|l|l|}
\hline Station & \multicolumn{1}{|c|}{ Bottom habitat } & \multicolumn{1}{|c|}{ locality } & depth \\
\hline 1 & Sludge/shells & out side the western harbor of Alexandria & $5 \mathrm{~m}$ \\
\hline 2 & Sludge/plant fragments & inside-El Mex Bay close to El Amom drain & $6 \mathrm{~m}$ \\
3 & Sludge/plant fragments & middle-El Mex Bey-El Amom drain & $12 \mathrm{~m}$ \\
4 & coarse sand & out side-El Mex Bay-El Amom drain & $10 \mathrm{~m}$ \\
\hline 5 & fine/coarse sand & close to Al Agamy beach,dense,urban area & $13 \mathrm{~m}$ \\
6 & coarse sand & out side Al Agamy beach & $14 \mathrm{~m}$ \\
\hline 7 & Sludge/plant fragments & close to El Nobaria drain & $2.5 \mathrm{~m}$ \\
8 & fine/coarse sand & out side El Nobaria drain & $4 \mathrm{~m}$ \\
9 & fine/coarse sand & out side El Nobaria drain & $9 \mathrm{~m}$ \\
10 & fine/coarse sand & out side El Nobaria drain & $12 \mathrm{~m}$ \\
\hline 11 & fine/calcareous sand & SUMED oil transport area, km 21 Sidi Kerier & $2 \mathrm{~m}$ \\
12 & fine/calcareous sand & SUMED oil transport area, km 21 Sidi Kerier & $25 \mathrm{~m}$ \\
\hline 13 & fine/calcareous sand & lies in between Sts. 12, 15 & $22 \mathrm{~m}$ \\
\hline 14 & fine/calcareous sand & in front Marakia resort beach & $2 \mathrm{~m}$ \\
15 & fine/calcareous sand & out side the area of Marakia resort & $20 \mathrm{~m}$ \\
\hline 16 & fine/calcareous sand & in front Marbilla resort beach & $2 \mathrm{~m}$ \\
\hline
\end{tabular}




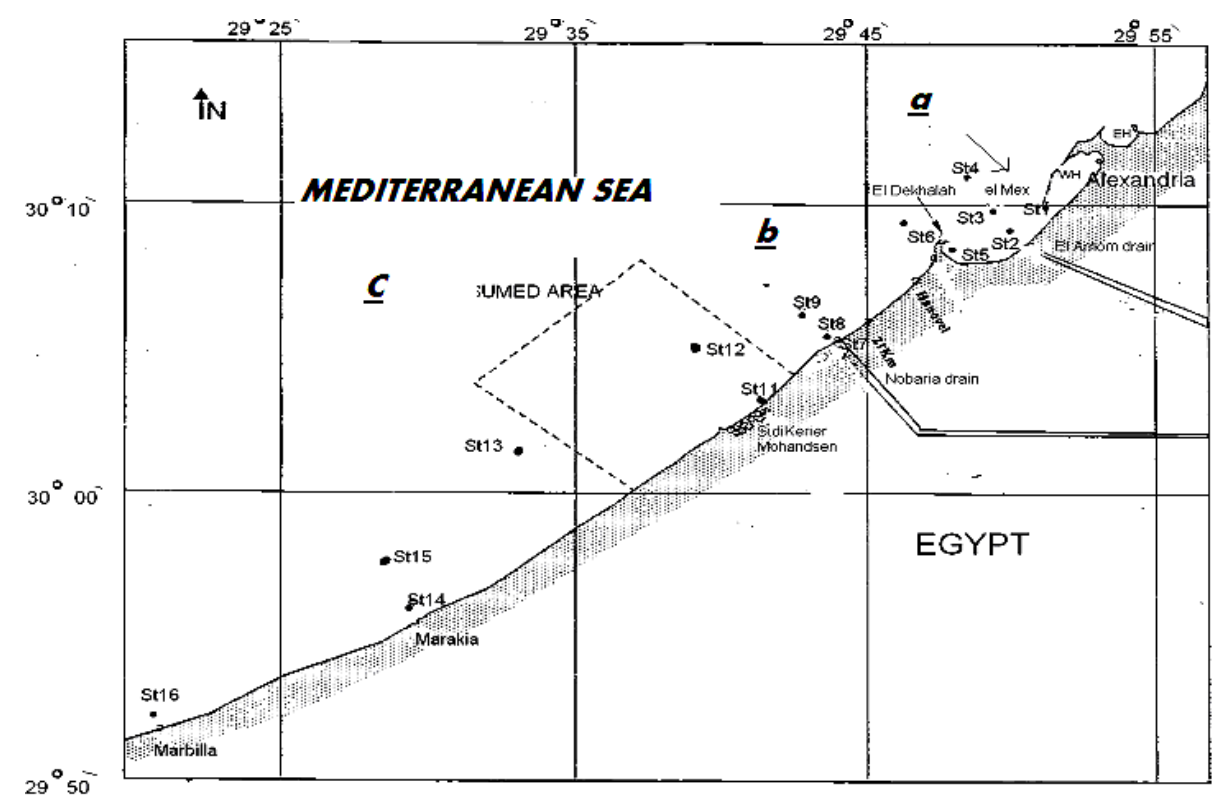

Fig. 1: Area of study and sampling stations, west of Alexandria.

\section{Fieldwork}

Bottom samples for benthic community analysis were taken bimonthly at 16 sites by using a grab bottom sampler sized $13 \times 18 \mathrm{~cm}$ (equivalent to $0.023 \mathrm{~m}^{2}$ ). The sampling was carried out during March 2005, May, July, September, November and January 2006. The bottom sediments sample at each site was partially sieved through a $500 \mathrm{um}$ sieve and the residue was preserved in containers with capacity one liter in $5-10 \%$ formalin for later taxonomic identification and enumeration.

\section{Laboratory work}

The sample was washed through a sieve with $0.2 \mathrm{~mm}$ and all sorted animals were identified to the lowest possible taxonomic level by microscopical investigation under a stereomicroscope $(20 \mathrm{x} 40 \mathrm{x})$. The numerical density of the bottom fauna was expressed as the number of individuals $/ \mathrm{m}^{2}$, while the corresponding wet weight was expressed as the wet weight in gram $/ \mathrm{m}^{2}$.

\section{Statistical Analysis}

Data analyses were carried out to calculate the diversity indices with Excel spread sheet and statistical analysis were performed with Minitab 14 and the Primer v5 package. These analyses were carried out on species composition structure of each site, the numerical number of benthos assemblages in addition to the biomass of the main fauna groups or species of benthos. The changes in the diversity of benthic structure over the time and sites were determined as follows:

a) The species diversity was calculated, using logarithms to the base ' $\mathrm{e}$ ' in the calculations, as the Shannon-Weaver (1949) index $\mathrm{H}^{\prime}=-\sum P i \log _{\mathrm{e}} P i$ where $P i=$ proportional of a sample of individuals belonging to species $i$

b) Pielou's Evenness index (1966) was calculated using the following equation: $J=$ $\mathrm{H}^{\prime} /$ loge S where S number of species.

c) Swartz's dominance index, which measured the number of species whose combined abundance; comprised $75 \%$ of the total sample abundance. According to PTI 1993, the values less than 5 usually indicate a stressed community (PTI, 1993 as cited by Laetz, 1998).

d) the analysis of variance (ANOVA) were computed by using simple linear regression between the variables at sampling sites (abundance, biomass, $\mathrm{H}^{\prime}$, number 
of species, main benthos groups) and physio-chemical variables of seawater and sediments ( $\mathrm{Ni}, \mathrm{Mn}, \mathrm{Fe}, \mathrm{Cu}, \mathrm{Zn}, \mathrm{Pb}, \mathrm{Cd}, \mathrm{NH}_{3}, \mathrm{NO}_{3}, \mathrm{NO}_{2}, \mathrm{PO}_{4}, \mathrm{SiO}_{4}, \mathrm{~S} \%$, temperature, $\mathrm{pH}$ as listed in the final Report, NIOF, 2006)

\section{RESULTS}

\section{Species composition}

At the different sampling sites, a great variation was noticed in the structure of bottom fauna as will as over the period of collections. The general structure of the bottom fauna is composed mainly from calcareous shells of molluscs, barnacles, and tubeworms. The living forms included mainly 18 groups and 80 species as listed in Table (1).

Table 1: List of species of macrobenthos recorded in the different sampling sites at the western coastal waters of Alexandria during March 2005 to January 2006.

\begin{tabular}{|c|c|c|}
\hline Algae: & Sedentary polychaetes & Decapoda \\
\hline Chaetomorpha aerea & Scolelepis ciliata & Portumnus latipes \\
\hline Halimedia tuna & Aonides oxycephala & Pagurus sp. \\
\hline Spatoglossum solierii & Polydora caeca & Sergestes vigilax \\
\hline Ectocarpus siliculosus & Prionospio cirrifera & Carcinus aestuali \\
\hline Coralina elongata & Magelona papillicornis & Perocessa edulis \\
\hline Ceramium rubrum & Cirratulus filiformis & Leptomysis mediterranean \\
\hline Ceramium cilliatum & Armandia polyophthalma & Mollusca \\
\hline Sea grass: & Capitella capitata & Gastropoda \\
\hline Posodonia oceanica & Owenia fusiformis & Patella nigra \\
\hline Sponges: & Sabella pavonina & Conus mediterranaeus \\
\hline Free living Nematoda: & Bispira volutacornis & Melanoides trunculita \\
\hline Bryozoa: & Crustacea : & Theodoxus niloticus \\
\hline Bugula neritina & Ostracoda : & Cypraea lurida \\
\hline Bugula turbinata & Amphipoda: & Nassarius mutabilis \\
\hline Schizoporella uniconis & Elasmopus pectenicurus & Diodora gibberula \\
\hline Bowerbankia gracilis & Gammarus aequicauda & Bivalves \\
\hline Bowerbankia imbricata & Hyale prevosta & Donax trunculus \\
\hline Annelida : & Meaera inaequipes & Donax venustus \\
\hline Oligochaeta : & Isopoda & Petricola lithophaga \\
\hline Errantia polychaetes & Arcturella dilotada & Dosinia exoleta \\
\hline Polygordius neapolitanus & Leptanthura tenuis & Modiolus adridicus \\
\hline Saccocirrus major & Sphaeroma walkeri & Modiolus barbatus \\
\hline Lepidonotus clava & Cirolana borealis & Cardium edule \\
\hline Polyodontes maxillosus & Anthura gracilis & Solemya togata \\
\hline Eulalia viridis & Idotea bultica & Loripes lucinalis \\
\hline Maupasia isochaeta & Anisopoda & Tellina pulchella \\
\hline Syllis gracilis & Apseudes latreillei & Sipunculida \\
\hline Nereis irrorata & Tanais cavolinii & Asoidosiphon muleri \\
\hline Nephthyis hombergii & Cummacea & Physcosoma vulgare \\
\hline Glycera convoluta & Pseudocuma longicornis & Ascidians \\
\hline Goniada norvegica & Cirripedia & Styela plicata \\
\hline Eunice vittata & Balanus perforatus & Cephalochordata \\
\hline Onuphis eremite & Balanus amphitrite & Amphiuxus lanceolatus \\
\hline Diopatra neapolitan & Balanus eburneus & \\
\hline Lumbriconereis laterilli & Balanus trigonus & \\
\hline
\end{tabular}

\section{Regional Distribution of macro benthos}

\section{Abundance}

It is more reliable to compare the numerical abundance and percentage showing different benthic community groups at the sampling sites of the study area. The regional distribution of benthic invertebrate's communities over period of collections indicated that the total abundance ranged from 959 to $8444 \mathrm{ind} . / \mathrm{m}^{2}$, with an annual 
average of 3072 ind. $/ \mathrm{m}^{2}$ as listed in Table (2) and graphically illustrated in Fig. 2. Seven predomination benthic groups of 18 groups are as following:

Table 2: Basic description variables for benthos samplings at the different sites along the western coastal waters of Alexandria during March 2005 to January 2006.

\begin{tabular}{|c|c|c|c|c|c|c|c|c|c|c|c|c|c|c|c|c|c|c|}
\hline & Parameters & St. 1 & St.2 & St. 3 & St. 4 & St. 5 & St. 6 & St. 7 & St. 8 & St. 9 & St. 10 & St. 11 & St. 12 & St. 13 & St. 14 & St. 15 & St. 16 & Total \\
\hline \multirow[t]{8}{*}{ Mar } & Abund. & 4578 & & 504 & 3612 & 1848 & 2016 & & 3444 & 1386 & 168 & 462 & & & 504 & & 1596 & 1829 \\
\hline & & $22.8 \%$ & & $2.5 \%$ & $18.0 \%$ & $9.2 \%$ & $10.0 \%$ & & $17.1 \%$ & $6.9 \%$ & $0.8 \%$ & $2.3 \%$ & & & $2.5 \%$ & & $7.9 \%$ & \\
\hline & Biomass & 1268 & & 0.01 & 37.4 & 3.4 & 100 & & 2.1 & 371 & 681 & 0.43 & & & 0.01 & & 190 & 241.2 \\
\hline & & $47.8 \%$ & & $0.00 \%$ & $1.4 \%$ & $0.1 \%$ & $3.8 \%$ & & $0.1 \%$ & $14.0 \%$ & $25.7 \%$ & $0.02 \%$ & & & $0.0 \%$ & & $7.2 \%$ & \\
\hline & No. sp. & 15 & & 1 & 5 & 4 & 6 & & 3 & 3 & 2 & 2 & & & 1 & & 4 & \\
\hline & $\begin{array}{c}\text { Swartz's } \\
\text { dominance }\end{array}$ & 6 & & 1 & 3 & 2 & 1 & & 1 & 1 & 2 & 1 & & & 1 & & 1 & \\
\hline & Evenness & 0.88 & & 0.00 & 0.84 & 0.82 & 0.62 & & 0.28 & 0.85 & 1.00 & 0.68 & & & 0.00 & & 0.67 & \\
\hline & $\mathrm{H}^{\prime} \log \mathrm{e}$ & 2.38 & & 0.00 & 1.36 & 1.14 & 1.12 & & 0.31 & 0.93 & 0.69 & 0.47 & & & 0.00 & & 0.93 & \\
\hline \multirow[t]{8}{*}{ May } & Abund. & 126 & 12390 & & 6888 & 10374 & 5418 & 7602 & 3486 & 4956 & 3906 & 2184 & & & 378 & & 882 & 4883 \\
\hline & & $0.2 \%$ & $21.1 \%$ & & $11.8 \%$ & $17.7 \%$ & $9.2 \%$ & $13.0 \%$ & $5.9 \%$ & $8.5 \%$ & $6.7 \%$ & $3.7 \%$ & & & $0.6 \%$ & $0.0 \%$ & $1.5 \%$ & \\
\hline & Biomass & 0.84 & 7.15 & & 71.4 & 13.9 & 163 & 158 & 1.27 & 6.73 & 326 & 27.3 & & & 95.34 & & 30.24 & 75.1 \\
\hline & & $0.1 \%$ & $0.8 \%$ & & $7.9 \%$ & $1.5 \%$ & $18.0 \%$ & $17.6 \%$ & $0.1 \%$ & $0.7 \%$ & $36.2 \%$ & $3.0 \%$ & & & $11 \%$ & $0.0 \%$ & $3.4 \%$ & \\
\hline & No. sp. & 2 & 5 & & 5 & 8 & 10 & 4 & 3 & 5 & 10 & 2 & & & 3 & & 4 & \\
\hline & $\begin{array}{c}\text { Swartz`s } \\
\text { dominance }\end{array}$ & 1 & 2 & & 1 & 1 & 3 & 2 & 1 & 3 & 4 & 1 & & & 2 & & 2 & \\
\hline & Evenness & 0.92 & 0.72 & & 0.45 & 0.49 & 0.74 & 0.76 & 0.24 & 0.80 & 0.81 & 0.32 & & & 0.88 & & 0.75 & \\
\hline & $\mathrm{H}^{\prime} \log \mathrm{e}$ & 0.64 & 1.17 & & 0.72 & 1.02 & 1.69 & 1.05 & 0.27 & 1.29 & 1.86 & 0.22 & & & 0.96 & & 1.05 & \\
\hline \multirow[t]{8}{*}{ Jul } & Abund. & 156 & & 588 & 6976 & 10406 & 5605 & 7768 & 3496 & 4978 & 4254 & 2219 & 0 & 0 & 482 & 0 & 927 & 3097 \\
\hline & & $0.3 \%$ & & $1.3 \%$ & $15.0 \%$ & $22.4 \%$ & $12.1 \%$ & $16.7 \%$ & $7.5 \%$ & $10.7 \%$ & $9.2 \%$ & $4.8 \%$ & $0.0 \%$ & $0.0 \%$ & $1.0 \%$ & $0.0 \%$ & $2.0 \%$ & \\
\hline & Biomass & 142.4 & & 0.01 & 1.69 & 10.1 & 527 & 405 & 1.69 & 2.53 & 3.78 & 0.01 & 1.69 & 812 & 0.85 & 0.85 & 0.43 & 127.3 \\
\hline & & $7.5 \%$ & & $0.0 \%$ & $0.09 \%$ & $0.53 \%$ & $27.6 \%$ & $21.2 \%$ & $0.09 \%$ & $0.13 \%$ & $0.20 \%$ & $0.00 \%$ & $0.09 \%$ & $42.5 \%$ & $0.0 \%$ & $0.04 \%$ & $0.02 \%$ & \\
\hline & No. sp. & 5 & & 1 & 4 & 7 & 8 & 5 & 7 & 8 & 6 & 1 & 5 & 4 & 3 & 3 & 2 & \\
\hline & $\begin{array}{c}\text { Swartz`s } \\
\text { dominance }\end{array}$ & 1 & & 1 & 1 & 2 & 3 & 2 & 3 & 3 & 3 & 1 & 2 & 2 & 2 & 1 & 1 & \\
\hline & Evenness & 0.42 & & 0.00 & 0.54 & 0.62 & 0.74 & 0.66 & 0.77 & 0.82 & 0.80 & 0.00 & 0.59 & 0.73 & 0.71 & 0.56 & 0.57 & \\
\hline & $\mathrm{H}^{\prime} \log \mathrm{e}$ & 0.76 & & 0.00 & 0.75 & 1.20 & 1.54 & 1.06 & 1.50 & 1.70 & 1.44 & 0.00 & 0.82 & 1.01 & 0.78 & 0.62 & 0.40 & \\
\hline
\end{tabular}

Table. 2. Cont.

\begin{tabular}{|c|c|c|c|c|c|c|c|c|c|c|c|c|c|c|c|c|c|c|}
\hline & Parameters & St. 1 & St.2 & St. 3 & St. 4 & St. 5 & St. 6 & St. 7 & St. 8 & St. 9 & St. 10 & St. 11 & St. 12 & St. 13 & St. 14 & St. 15 & St. 16 & Total \\
\hline \multirow[t]{8}{*}{ Sep } & Abund. & 4578 & 1470 & 3234 & 546 & 966 & 1092 & 714 & 1596 & 3612 & 9114 & 756 & & & 1218 & & 588 & 2268 \\
\hline & & $\begin{array}{l}15.5 \\
\%\end{array}$ & $5.0 \%$ & $\begin{array}{l}11.0 \\
\%\end{array}$ & $1.8 \%$ & $3.3 \%$ & $3.7 \%$ & $2.4 \%$ & $5.4 \%$ & $12.2 \%$ & $30.9 \%$ & $2.6 \%$ & & & $4.1 \%$ & & $2.0 \%$ & \\
\hline & Biomass & 126.4 & 0.8 & 47.5 & 0.4 & 2.5 & 538 & 45.4 & 14.3 & 444 & 3068 & 1.7 & & & 2.5 & & 0.9 & 330.2 \\
\hline & & $2.9 \%$ & $0.02 \%$ & $1.1 \%$ & $\begin{array}{l}0.01 \\
\%\end{array}$ & $0.1 \%$ & $12.5 \%$ & $1.1 \%$ & $0.3 \%$ & $10.4 \%$ & $71.5 \%$ & $0.04 \%$ & & & $0.1 \%$ & & $0.02 \%$ & \\
\hline & No. sp. & 9 & 2 & 10 & 2 & 3 & 4 & 3 & 6 & 12 & 9 & 4 & & & 6 & & 2 & \\
\hline & $\begin{array}{l}\begin{array}{l}\text { Swartz's } \\
\text { dominance }\end{array} \\
\end{array}$ & 4 & 2 & 2 & 1 & 2 & 2 & 2 & 3 & 5 & 3 & 3 & & & 2 & & 1 & \\
\hline & Evenness & 0.82 & 0.89 & 0.58 & 0.62 & 0.84 & 0.87 & 0.87 & 0.82 & 0.84 & 0.66 & 0.91 & & & 0.74 & & 0.59 & \\
\hline & $\mathrm{H}^{`} \log \mathrm{e}$ & 1.80 & 0.97 & 1.32 & 0.43 & 0.92 & 1.20 & 0.96 & 1.47 & 2.09 & 1.46 & 1.26 & & & 1.33 & & 0.41 & \\
\hline \multirow[t]{8}{*}{ Nov } & Abund. & 1596 & 11466 & 756 & 1512 & 3780 & 2982 & 6636 & 3486 & 4662 & 2520 & 8022 & & & 4746 & & 1176 & 4103 \\
\hline & & $3.0 \%$ & $21.5 \%$ & $1.4 \%$ & $2.8 \%$ & $7.1 \%$ & $5.6 \%$ & $12.4 \%$ & $6.5 \%$ & $8.7 \%$ & $4.7 \%$ & $15.0 \%$ & & & $8.9 \%$ & & $2.2 \%$ & \\
\hline & Biomass & 1.7 & 8.8 & 0.9 & 1.7 & 390 & 2.1 & 276 & 1.3 & 6.3 & 5.5 & 5.5 & & & 2.5 & & 364 & 82.0 \\
\hline & & $0.2 \%$ & $0.8 \%$ & $0.1 \%$ & $0.2 \%$ & $36.6 \%$ & $0.2 \%$ & $25.8 \%$ & $0.1 \%$ & $0.6 \%$ & $0.5 \%$ & $0.5 \%$ & & & $0.2 \%$ & & $34.2 \%$ & \\
\hline & No. sp. & 3 & 5 & 2 & 3 & 13 & 3 & 3 & 2 & 11 & 10 & 2 & & & 2 & & 2 & \\
\hline & $\begin{array}{l}\text { Swartz's } \\
\text { dominance }\end{array}$ & 1 & 1 & 1 & 1 & 5 & 1 & 2 & 1 & 4 & 2 & 1 & & & 1 & & 1 & \\
\hline & Evenness & 0.40 & 0.37 & 0.50 & 0.76 & 0.80 & 0.68 & 0.83 & 0.98 & 0.77 & 0.81 & 0.80 & & & 0.92 & & 0 & \\
\hline & $\mathrm{H}^{\prime} \log \mathrm{e}$ & 0.28 & 0.60 & 0.35 & 0.53 & 2.05 & 0.74 & 0.91 & 0.68 & 1.85 & 1.30 & 0.55 & & & 0.64 & & 0.00 & \\
\hline \multirow[t]{7}{*}{ Jan } & Abund. & 2268 & & 1008 & 3150 & 2646 & 7770 & 840 & 1260 & 3906 & 5292 & 3990 & 546 & 420 & 1638 & & 588 & 2523 \\
\hline & & $6.4 \%$ & & $2.9 \%$ & $8.9 \%$ & $7.5 \%$ & $22.0 \%$ & $2.4 \%$ & $3.6 \%$ & $11.1 \%$ & $15.0 \%$ & $11.3 \%$ & $1.5 \%$ & $1.2 \%$ & $4.6 \%$ & & $1.7 \%$ & \\
\hline & Biomass & 2.1 & & 0.9 & 23.1 & 213.8 & 699.7 & 85.7 & 57.6 & 1.7 & 43.7 & 1.7 & 355.7 & 1.3 & 0.4 & & 0.01 & 106.2 \\
\hline & & $0.1 \%$ & & $0.1 \%$ & $1.6 \%$ & $14.4 \%$ & $47.1 \%$ & $5.8 \%$ & $3.9 \%$ & $0.1 \%$ & $2.9 \%$ & $0.1 \%$ & $23.9 \%$ & $0.1 \%$ & $0.0 \%$ & & $0.0 \%$ & \\
\hline & No. sp. & 4 & & 3 & 10 & 8 & 15 & 3 & 6 & 8 & 13 & 4 & 4 & 3 & 2 & & 1 & \\
\hline & $\begin{array}{l}\text { Swartz`s } \\
\text { dominance }\end{array}$ & 2 & & 1 & 5 & 4 & 6 & 1 & 2 & 4 & 6 & 1 & 1 & 2 & 1 & & 1 & \\
\hline & Evenness & 0.88 & & 0.59 & 0.91 & 0.83 & 0.83 & 0.95 & 0.91 & 0.81 & 0.88 & 0.64 & 0.81 & 0.99 & 0.68 & & 0 & \\
\hline
\end{tabular}




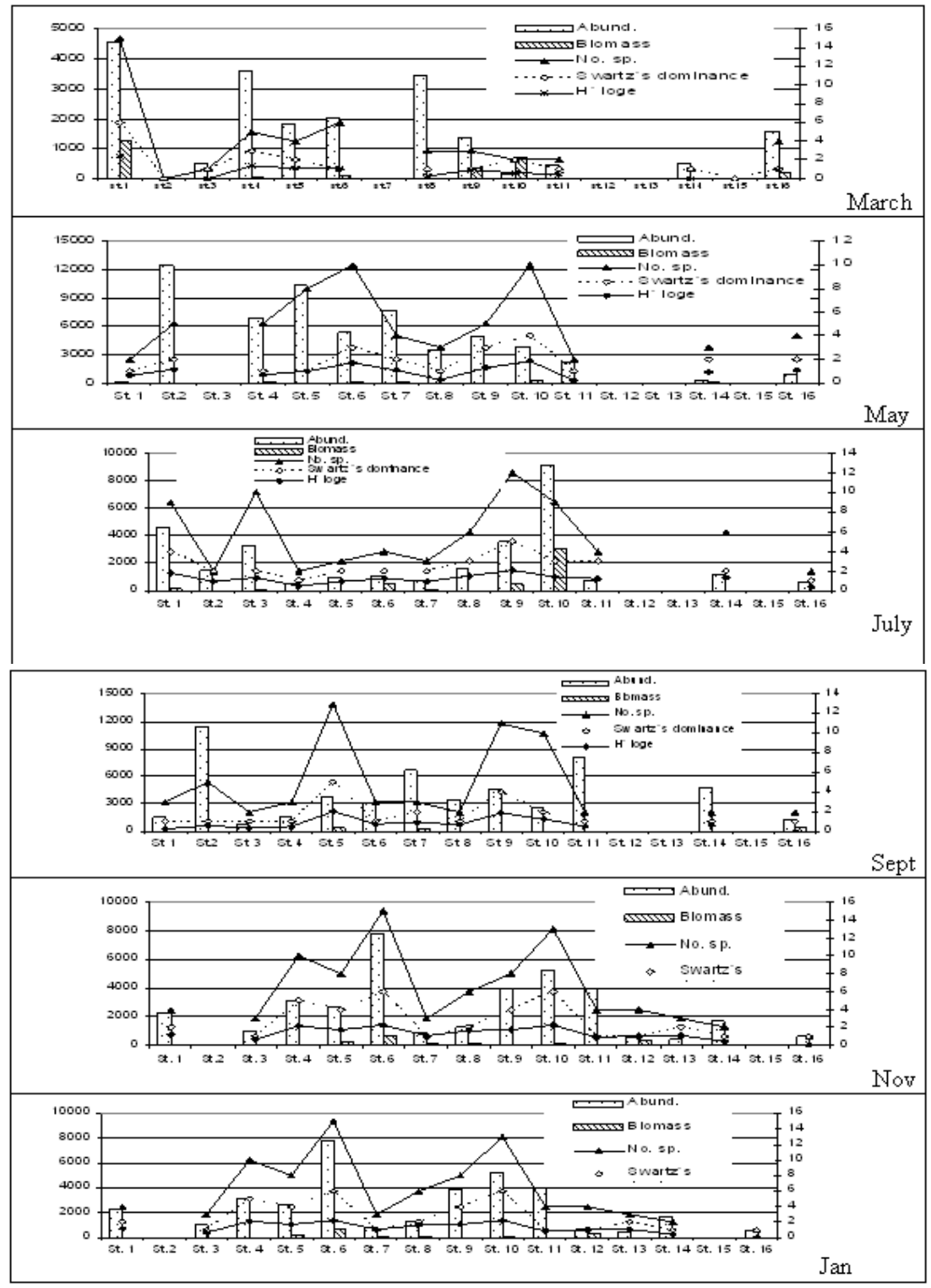

Fig. 2: Average annual density, biomass, Swartz`s abundance and number of species and diversity index of benthos communities at different sampling sites at the western coastal waters of Alexandria, during March 2005 to January 2006.

-Free-living nematodes; the dominant benthic group at all sites, representing $43.6 \%$ of the total benthic numerical abundance, was minimal of $336 \mathrm{ind} . / \mathrm{m}^{2}$ at site 16 , moderate 500-1000 ind. $/ \mathrm{m}^{2}$ at sites 5, 10, 12 and maximal $1000-2500 \mathrm{ind} . / \mathrm{m}^{2}$ at the rest of sites in the eutrophic area close to pollutant outfalls (El Amom and Nobaria drains).

-Oligochaetes; were recorded at most sites except at sites $8,12,15$, representing only $4 \%$, but highly recorded at sites $1,2,5,7,9,14$ that varied from $100-500 \mathrm{ind} . / \mathrm{m}^{2}$.

-Polychaetes; the $2^{\text {nd }}$ dominant benthic group and were found at all sites, representing $22 \%$, predominated at high eutrophic area close to the outfalls (sites 1, 2, 4, 5, 7, 8, 9, $10,11,13$ ) attaining $500-1200 \mathrm{ind} . / \mathrm{m}^{2}$, and the density dropped to $200-500 \mathrm{ind} . / \mathrm{m}^{2}$, at the rest of sites.

-Amphipods; the third common benthic group, representing 13.8\%, observed at most sites, dominated at site 2 close to El Amom drain with low salinity, yielding 5350 ind. $/ \mathrm{m}^{2}$. At sites $1,6,9$ the density reached $200-400$ ind. $/ \mathrm{m}^{2}$, dropped to $100-150$ ind. $/ \mathrm{m}^{2}$ at sites 14,10 , and the lowest density was at sites 8,16 with $20-50$ ind. $/ \mathrm{m}^{2}$. 
-Cirripedes, representing 4.8\%, predominated at site 1 (Western Harbour), sites 5, 6 (Dekhela harbour) and site 10 (out side Nobaria drain) ranging from 200 to 1400 ind. $/ \mathrm{m}^{2}$ and least abundant at sites 4, 9 (25-100 ind. $\left./ \mathrm{m}^{2}\right)$.

-Gastropods; representing $3.0 \%$ being maximal at site 7 close to El Nobaria drain, reaching $1220 \mathrm{ind} . / \mathrm{m}^{2}$, moderate at site $1\left(200 \mathrm{ind} . / \mathrm{m}^{2}\right)$, and with little numbers (7-30 ind. $/ \mathrm{m}^{2}$ ) at sites $5,10,14$.

-Bivalves; representing only $1.5 \%$, relatively high at site 16 , attaining 400 ind. $/ \mathrm{m}^{2}$ and, ranged from 50-100 ind. $/ \mathrm{m}^{2}$ at sites $1,5,8,10$ and the least density was at sites 4, 6, 12 (20-30 ind. $\left./ \mathrm{m}^{2}\right)$.

The abundance of macro benthos organisms at different sampling sites in the seawater ranked in the following sequence: Nematodes $46 \%>$ polychaetes $22 \%>$ amphipods $13.8 \%>$ cirripedes $4.8 \%>$ oligochaetes $4 \%>$ gastropods $3.0 \%>$ bivalves $1.5 \%$. In general, the abundance of macro-benthos organisms at the areas in front of the two main drains namely El Amom (sites 2, 3, 4) and Nobaria (sites 7, 8, 9, 10) contributed the greatest annual average. The average abundance of benthos at the former drain attained $4373 \mathrm{ind} . / \mathrm{m}^{2}$ and at the latter drain reaching, $3542 \mathrm{ind} . / \mathrm{m}^{2}$, representing respectively $26.5 \%$ and $29 \%$ of the total annual average of benthos at the study area, whereas the corresponding average biomass yielded $12.5 \mathrm{~g} / \mathrm{m}^{2}(1.4 \%)$ at El Amom area and $256 \mathrm{~g} / \mathrm{m}^{2}(41.7 \%)$ at Nobaria area.

\section{Biomass}

The biomass of algae and living calcareous forms as barnacles, gastropods, bivalves, and polychaetes showed the largest intensity. Biomass of benthic communities revealed significant differences at sampling sites as shown in Table (3).

The general biomass of benthic structure over the period of collections indicated that the total wet weight varied widely from one gram to $1172 \mathrm{~g} / \mathrm{m}^{2}$ with an annual average of $155 \mathrm{~g} / \mathrm{m}^{2}$. Six predominant benthic groups of the 17 groups are as following:

-Algae; the dominant benthic group at most sites, representing $30.6 \%$ of the total benthic biomass, being maximal $\left(180-400 \mathrm{~g} / \mathrm{m}^{2}\right)$ at the least stressed sites 12,16 , then moderate $\left(30-80 \mathrm{~g} / \mathrm{m}^{2}\right)$ at sites $1,6,9$ and least density at sites 4,16 weighing 2-15 $\mathrm{g} / \mathrm{m}^{2}$.

-Sea grass; representing 7.0\%, yielding $75-90 \mathrm{~g} / \mathrm{m}^{2}$ at Nobaria area sites 6,9 and varies from $1-5 \mathrm{~g} / \mathrm{m}^{2}$ at sites $11,14,16$.

-Polychaetes; less dense (1.7\%) yielding $10-11 \mathrm{~g} / \mathrm{m}^{2}$ at sites 5,7 and less than $3 \mathrm{~g} / \mathrm{m}^{2}$ at the rest of sites.

-Cirripedes; the dominant benthic group, representing $33.5 \%$ of the total benthic biomass, being maximal $\left(550 \mathrm{~g} / \mathrm{m}^{2}\right)$ at site 10 , moderate at site 6 (attaining $125 \mathrm{~g} / \mathrm{m}^{2}$ ), ranged from $50-100 \mathrm{~g} / \mathrm{m}^{2}$ at sites 1,5 and reached $16 \mathrm{~g} / \mathrm{m}^{2}$ at site 9 .

-Gastropods; representing $12.7 \%$, being maximal at site 7 close to Nobaria outfalls (yielded $\left.183 \mathrm{~g} / \mathrm{m}^{2}\right)$, moderate at site $10\left(113 \mathrm{~g} / \mathrm{m}^{2}\right)$, with little biomass $\left(5-14 \mathrm{~g} / \mathrm{m}^{2}\right)$ at sites $5,14$.

-Bivalves; representing 9.1\%, being relatively high at site $1,16\left(70-100 \mathrm{~g} / \mathrm{m}^{2}\right)$ and, ranged from $10-20 \mathrm{~g} / \mathrm{m}^{2}$ at sites $4,5,8$ and the least biomass was at sites $6,10,12(1-7$ $\left.\mathrm{g} / \mathrm{m}^{2}\right)$.

The biomass of benthic communities at different sampling sites ranked in the following sequence: Cirripeds 33.5\% > Algae 30.6\% > Gastropods 12.7\% > Bivalves $9.1 \%>$ Sea grass $7.0 \%>$ Polychaetes $1.7 \%$. During May and November, the average benthos sustained relatively high density reaching $4883 \mathrm{ind} . / \mathrm{m}^{2}$ and representing $24 \%$ of total numerical abundance of benthos with only $9 \%$ of total average biomass 136 
$\mathrm{g} / \mathrm{m}^{2}$ while the highest biomass was recorded in September $(66 \%$ with biomass and $11 \%$ with account number).

Table 3: Regional distribution of macrobenthos groups density (no. of individual $/ \mathrm{m}^{2}$ ) and biomass $\left(\mathrm{g} / \mathrm{m}^{2}\right)$ at the different sites along the western coastal waters of Alexandria during March 2005

to January 2006.

\begin{tabular}{|c|c|c|c|c|c|c|c|c|c|c|c|c|c|c|c|c|c|c|}
\hline Abundance & St1 & St2 & St3 & St4 & St5 & St6 & St7 & St8 & St9 & St10 & St11 & St12 & St13 & St14 & St15 & St16 & average & $\%$ \\
\hline Algae & 105 & 0 & 0 & 28 & 0 & 308 & 0 & 0 & 175 & 364 & 0 & 126 & 63 & 35 & 0 & 42 & 77.9 & $2.5 \%$ \\
\hline Sea grass & 0 & 0 & 0 & 0 & 0 & 56 & 0 & 0 & 42 & 0 & 21 & 0 & 0 & 28 & 0 & 7 & 9.6 & $0.3 \%$ \\
\hline Sponges & 0 & 0 & 0 & 0 & 0 & 14 & 0 & 0 & 0 & 0 & 0 & 0 & 0 & 0 & 0 & 0 & 0.9 & $0.0 \%$ \\
\hline Nematoda & 1743 & 2002 & 949 & 2065 & 2499 & 1841 & 1344 & 1862 & 1575 & 714 & 1372 & 903 & 735 & 1050 & 1596 & 336 & 1411.6 & $46.0 \%$ \\
\hline Bryozoa & 126 & 0 & 42 & 0 & 0 & 0 & 0 & 0 & 0 & 14 & 0 & 0 & 0 & 0 & 0 & 0 & 11.4 & $0.4 \%$ \\
\hline Oligochaeta & 56 & 448 & 34 & 49 & 245 & 56 & 202 & 0 & 280 & 98 & 91 & 0 & 63 & 147 & 0 & 84 & 121.9 & $4.0 \%$ \\
\hline Polychaeta & 672 & 644 & 160 & 1078 & 1078 & 441 & 949 & 931 & 1106 & 833 & 1218 & 441 & 504 & 455 & 378 & 21 & 675.7 & $22.0 \%$ \\
\hline Ostracoda & 0 & 0 & 0 & 0 & 84 & 42 & 0 & 0 & 119 & 0 & 0 & 0 & 0 & 0 & 0 & 0 & 15.3 & $0.5 \%$ \\
\hline Amphipoda & 329 & 5350 & 25 & 147 & 0 & 287 & 0 & 42 & 406 & 126 & 0 & 0 & 0 & 0 & 0 & 49 & 422.6 & $13.8 \%$ \\
\hline Isopoda & 56 & 0 & 0 & 28 & 0 & 56 & 17 & 14 & 0 & 77 & 0 & 0 & 0 & 7 & 0 & 0 & 15.9 & $0.5 \%$ \\
\hline Anisopoda & 28 & 0 & 0 & 0 & 0 & 84 & 0 & 0 & 21 & 21 & 0 & 0 & 0 & 0 & 0 & 0 & 9.6 & $0.3 \%$ \\
\hline Cirripedia & 231 & 0 & 0 & 35 & 224 & 392 & 0 & 0 & 98 & 1358 & 0 & 0 & 0 & 0 & 0 & 0 & 146.1 & $4.8 \%$ \\
\hline Decapoda & 0 & 0 & 8 & 0 & 7 & 0 & 0 & 0 & 0 & 7 & 0 & 0 & 0 & 7 & 0 & 21 & 3.2 & $0.1 \%$ \\
\hline Cummacea & 0 & 0 & 0 & 0 & 0 & 0 & 0 & 0 & 21 & 0 & 0 & 0 & 0 & 0 & 0 & 0 & 1.3 & $0.0 \%$ \\
\hline Gastropoda & 203 & 0 & 0 & 0 & 28 & 0 & 1218 & 0 & 0 & 14 & 0 & 0 & 0 & 7 & 0 & 0 & 91.9 & $3.0 \%$ \\
\hline Bivalves & 91 & 0 & 0 & 28 & 70 & 21 & 0 & 49 & 0 & 70 & 0 & 21 & 0 & 0 & 0 & 399 & 46.8 & $1.5 \%$ \\
\hline Sipunculida & 0 & 0 & 0 & 14 & 0 & 119 & 0 & 0 & 0 & 0 & 0 & 0 & 0 & 0 & 0 & 0 & 8.3 & $0.3 \%$ \\
\hline Ascidians & 21 & 0 & 0 & 0 & 0 & 0 & 0 & 0 & 0 & 0 & 0 & 0 & 0 & 0 & 0 & 0 & 1.3 & $0.0 \%$ \\
\hline $\begin{array}{l}\text { Amphioxus } \\
\text { lanceolatus }\end{array}$ & 0 & 0 & 0 & 0 & 0 & 0 & 0 & 0 & 0 & 0 & 0 & 21 & 0 & 0 & 0 & 0 & 1.3 & $0.0 \%$ \\
\hline Abundance & 3661 & 8444 & 1218 & 3472 & 4235 & 3717 & 3730 & 2898 & 3843 & 3696 & 2702 & 1512 & 1365 & 1736 & 1974 & 959 & 3073 & $100 \%$ \\
\hline$\%$ & $7.4 \%$ & $17.2 \%$ & $2.5 \%$ & $7.1 \%$ & $8.6 \%$ & $7.6 \%$ & $7.6 \%$ & $5.9 \%$ & $7.8 \%$ & $7.5 \%$ & $5.5 \%$ & $3.1 \%$ & $2.8 \%$ & $3.5 \%$ & $4.0 \%$ & $2.0 \%$ & $100 \%$ & \\
\hline Biomass & St1 & St2 & St3 & St4 & St5 & $\begin{array}{l}\text { St6 } \\
\end{array}$ & St7 & St8 & St9 & St10 & St11 & St.12 & St.13 & $\begin{array}{l}\text { St.14 } \\
\end{array}$ & $\begin{array}{l}\text { St.15 } \\
\end{array}$ & $\begin{array}{l}\text { St.16 } \\
\end{array}$ & average & $\%$ \\
\hline Algae & 29 & 0 & 0 & 0 & 0 & 81 & 0 & 0 & 46 & 15 & 0 & 176 & 406 & 5.8 & 0 & 1.6 & 47.5 & $30.6 \%$ \\
\hline Sea grass & 0 & 0 & 0 & 0 & 0 & 90 & 0 & 0 & 74 & 0 & 4.6 & 0 & 0 & 5.1 & 0 & 1.2 & 10.9 & $7.0 \%$ \\
\hline Sponges & 0 & 0 & 0 & 0 & 0 & 22 & 0 & 0 & 0 & 0 & 0 & 0 & 0 & 0 & 0 & 0 & 1.4 & $0.9 \%$ \\
\hline Nematoda & 0 & 0 & 0 & 0 & 0 & 0 & 0 & 0 & 0 & 0 & 0 & 0 & 0 & 0 & 0 & 0 & 0.0 & $0.0 \%$ \\
\hline Bryozoa & 1.1 & 0 & 0 & 0 & 0 & 0 & 0 & 0 & 0 & 0 & 0 & 0 & 0 & 0 & 0 & 0 & 0.1 & $0.1 \%$ \\
\hline Oligochaeta & 0 & 0.6 & 0 & 0 & 0 & 0 & 0 & 0 & 0 & 0 & 0 & 0 & 0 & 0 & 0 & 0 & 0.2 & $0.1 \%$ \\
\hline Polychaeta & 1.9 & 2.1 & 0.4 & 3.2 & 10.8 & 1.5 & 10.4 & 1.5 & 2.6 & 1.9 & 1.3 & 1.3 & 0.6 & 0.5 & 0.8 & 0 & 2.6 & $1.7 \%$ \\
\hline Ostracoda & 0 & 0 & 0 & 0 & 0 & 0 & 0 & 0 & 0 & 0 & 0 & 0 & 0 & 0 & 0 & 0 & 0.0 & $0.0 \%$ \\
\hline Amphipoda & 0 & 3 & 0 & 0 & 0 & 0 & 0 & 0 & 0 & 0 & 0 & 0 & 0 & 0 & 0 & 0 & 0.3 & $0.2 \%$ \\
\hline Isopoda & 0 & 0 & 0 & 0 & 0 & 0 & 0 & 0 & 0 & 0 & 0 & 0 & 0 & 0 & 0 & 0 & 0.0 & $0.0 \%$ \\
\hline Anisopoda & 0 & 0 & 0 & 0 & 0 & 0 & 0 & 0 & 0 & 0 & 0 & 0 & 0 & 0 & 0 & 0 & 0.0 & $0.0 \%$ \\
\hline Cirripedia & 83 & 0 & 0 & 0 & 56 & 126 & 0 & 0 & 16 & 550 & 0 & 0 & 0 & 0 & 0 & 0 & 52.0 & $33.5 \%$ \\
\hline Decapoda & 0 & 0 & 7.3 & 0 & 5.0 & 0 & 0 & 0 & 0 & 0 & 0 & 0 & 0 & 0 & 0 & 26 & 2.4 & $1.6 \%$ \\
\hline Cummacea & 0 & 0 & 0 & 0 & 0 & 0 & 0 & 0 & 0 & 0 & 0 & 0 & 0 & 0 & 0 & 0 & 0.0 & $0.0 \%$ \\
\hline Gastropoda & 0 & 0 & 0 & 0 & 14 & 0 & 183 & 0 & 0 & 113 & 0 & 0 & 0 & 5.0 & 0 & 0 & 19.7 & $12.7 \%$ \\
\hline Bivalves & 94 & 0 & 0 & 16 & 19 & 7.2 & 0 & 11 & 0 & 7.1 & 0 & 1.1 & 0 & 0 & 0 & 69 & 14.1 & $9.1 \%$ \\
\hline Sipunculida & 0 & 0 & 0 & 2.8 & 0 & 10 & 0 & 0 & 0 & 0 & 0 & 0 & 0 & 0 & 0 & 0 & 0.8 & $0.5 \%$ \\
\hline Ascidians & 46 & 0 & 0 & 0 & 0 & 0 & 0 & 0 & 0 & 0 & 0 & 0 & 0 & 0 & 0 & 0 & 2.9 & $1.9 \%$ \\
\hline $\begin{array}{l}\text { Amphioxus } \\
\text { lanceolatus }\end{array}$ & 0 & 0 & 0 & 0 & 0 & 0 & 0 & 0 & 0 & 0 & 0 & 0 & 0 & 0 & 0 & 0 & 0.0 & $0.0 \%$ \\
\hline $\begin{array}{c}\text { Biomass } \\
\mathrm{g} / \mathrm{m} 2\end{array}$ & 257 & 6 & 8 & 23 & 106 & 338 & 194 & 13 & 139 & 688 & 6 & 180 & 407 & 17 & 1 & 98 & 155 & $100 \%$ \\
\hline$\%$ & $10.4 \%$ & $0.2 \%$ & $0.3 \%$ & $0.9 \%$ & $4.3 \%$ & $13.6 \%$ & $7.8 \%$ & $0.5 \%$ & $5.6 \%$ & $27.7 \%$ & $0.2 \%$ & $7.3 \%$ & $16.4 \%$ & $0.7 \%$ & $0.0 \%$ & $3.9 \%$ & $100 \%$ & \\
\hline No. sp. & 6.3 & 4.0 & 3.4 & 4.8 & 7.2 & 7.7 & 3.6 & 4.5 & 7.8 & 8.3 & 2.5 & 4.5 & 3.5 & 2.8 & 3.0 & 2.5 & & \\
\hline $\mathrm{H}^{\prime}$ & 1.64 & 1.26 & 0.62 & 1.44 & 1.94 & 1.98 & 1.45 & 1.40 & 2.30 & 2.17 & 0.82 & 1.40 & 1.51 & 1.01 & 0.89 & 0.78 & & \\
\hline
\end{tabular}




\section{Species number (S)}

At the different sites, the general structure of species number of benthos groups emphasized that Polychaeta were the most common, comprising 26 species of a total number of 80 taxa as shown in Table (1). It was followed by Bivalvia (10 species), algae and gastropods 7 species, isopods and decapods 6 species, bryozoans 5 species, amphipods 4 species, and cirripedes 4 species. As shown in Table (2) and Fig. 2, the highest number of species was 15 at sites 1, 6 in March and January, 13 species at sites 5, 10 in November and January and 12 species at site 9 in September. At most sites over time, the number of species varied from one to 10 species.

\section{Species diversity $\left(\mathrm{H}^{\prime}\right)$}

In general, species diversity index was relatively high in particular at site 1 in March, at sites $6 \& 10$ in May, at sites $9 \& 6$ in July, at sites $1 \& 9$ in September, at sites $5 \& 9$ in November and at sites 4, 6, 10 in January as shown in Table (2) and Fig. 2. The estimated values of the species composition diversity at the study area revealed that the high species diversity of benthos is greatly related to the number of species and numerical abundance of individuals. The values of species diversity $\left(\mathrm{H}^{\prime}\right)$ were relatively moderate $(1.3-2.02)$ at most stations with low diversity at site $7(0.9)$.

On the other hand, the species diversity was only 1.6 at site 2 though it was the richest which might be attributed to the number of species, which the dropping of diversity is primarily attributed to the uneven distribution of individuals among species. The general picture of the benthos community's diversity at the sampling sites by using the total average benthic groups as shown in Fig 3, illustrating that sites $1,6,9$, and 10 show high diversity.

\section{Evenness ( $\mathbf{J})$ and Richness (d)}

Values of evenness and richness indices were significantly high only at sites 3 , 14, 16 (Table 2). As shown in Fig. 3, these values were calculated by using the annual average data which revealed that the richness was high $(>1)$ at sites 1, 9, 10, 6 while the evenness value index was about 0.8 at only site 7 .

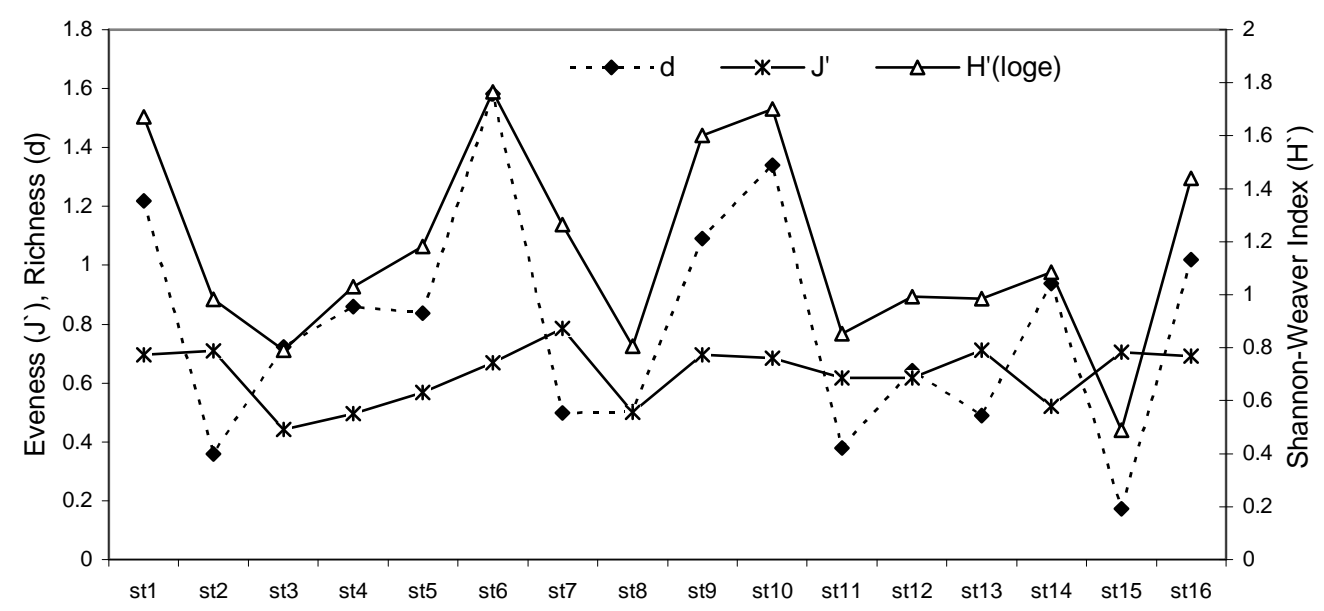

Fig.3: Diversity indices of the average annual density of main benthic groups at different sampling sites at the western coastal waters of Alexandria, during March 2005 to January 2006.

\section{Swartz's index}

Swartz's dominance index, which measured the number of species whose combined abundance comprised $75 \%$ of the total sample abundance (if index value $>$ 5 indicating that the area has stress community). As shown in Table (2) the Swartz's dominance index values ranged from one to six at the sampling sites, where most 
western sites were significantly of less stress than the previous sites. Site 1 in March, site 9 in September, site 5 in November and sites 4, 6, 10 in January were relatively of less stress with high diversity and number of species.

\section{Abundance-Sites and diversity index Clustering}

Dendrograms based on Euclidean distances for clustering of 16 numerical density variables of all benthos collected at the different sites is shown in Fig. 3B.

The highest similarity was noticed between the group site 5 and one class includes sites 4, 8. Group 2 includes two sites 1, 6. Group 3 includes three sites 12, 13,15 , where the remaining sites were of low similarity.

Fig. 3A indicates the clustering analysis of the diversity indices of benthic communities at different sites, indicating that diversity $\mathrm{H}^{\prime}$ index is clearly influenced by number of species, richness $(d)$ index and evenness $\left(J^{\prime}\right)$ index. The similarity of benthic groups (Fig. 3C) was high between polycheates and nematodes.

A

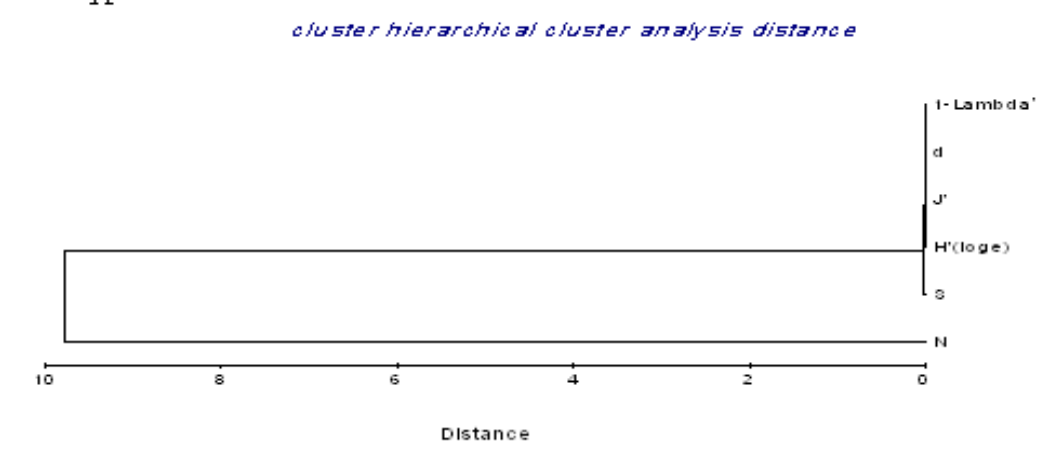

B

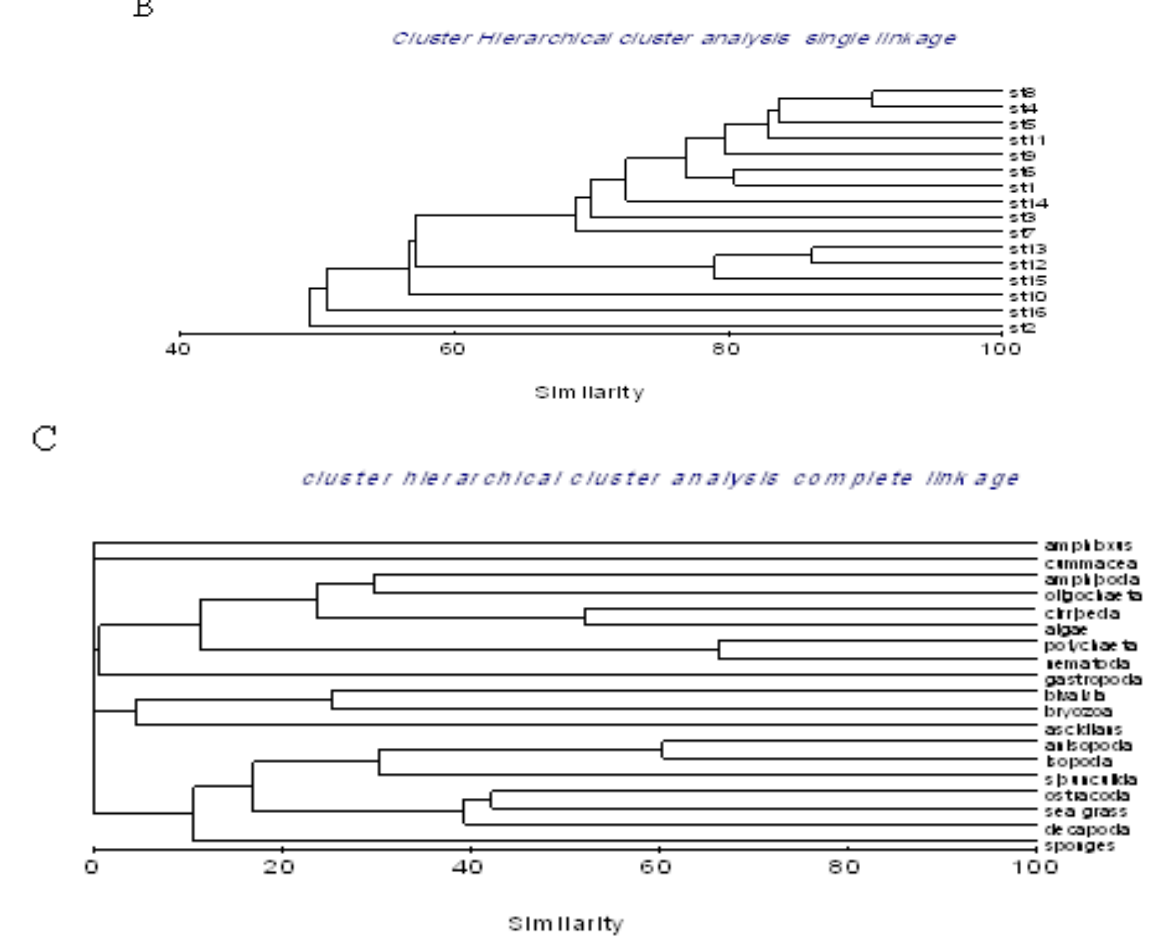

Fig. 4: Diagram for diversity indices and abundance of macrobenthos groups at sampling sites in the western coastal waters of Alexandria, during March 2005 to January 2006. 


\section{Linear regression analysis}

Statistical analysis showed significant correlation between some of the chemical parameters in sediment and seawater with the data of the bottom fauna. Square matrix at the $95 \%$ confidence was used. Pearson correlation coefficient $(r)$ at $\mathrm{P}$ level $<0.05$ measuring the significance of the various environmental parameters are shown in details in Table (4) that indicates that the following correlation's were significant ( $\mathrm{P}$ $<0.05$ );

- The abundance of Sponges shows significant and positive correlation with the content of dissolved $\mathrm{Ni}$ in the water column.

- The abundance of Nematodes appears significant and positive correlation with the content of Mn in the water column. It had negative correlation content of $\mathrm{Ni}$ in sediments.

- The abundance of Amphipods shows negative correlation to the value of $\mathrm{pH}$ of seawater but shows positive correlation to the content of $\mathrm{NH}_{3}, \mathrm{NO}_{2}, \mathrm{SiO}_{4}$, in water column.

- The abundance of Anisopoda correlated positively with the content of $\mathrm{Mn}$ in water.

- The abundance of Decapods shows positive correlation to concentration of $\mathrm{Mn}$ in sea water column.

- The abundance of Gastropods appears significant with negative correlation with the degree of seawater temperature and salinity. However, it showed significantly positive correlation with the $\mathrm{NO}_{3}, \mathrm{PO}_{4}, \mathrm{SiO}_{4}$.

- The abundance of Bivalves indicates significant positive correlation with the depth of water column and the oxygen content of seawater.

- The abundance of Sipunculida shows significantly positive correlation with the concentration of $\mathrm{Mn}$ in water column.

- The abundance of benthos indicates significantly negative correlation with $\mathrm{Ni}$ in sediments.

- The number of benthos species shows positive correlation with degree of seawater temperature.

Table 4: Correlation between the benthic groups and the prevailing hydrographical arameters of the coastal waters and sediments at the sampling sites along the western coastal waters of Alexandria during March 2005 to January 2006.

$\mathrm{r}=$ Pearson correlation coefficient (marked correlations are significant at $\mathrm{p}<0.005$

\begin{tabular}{|l|l|l|l|l|l|l|l|l|l|l|l|l|l|l|l|}
\hline & Depth & Temp & $\mathrm{S} \% 0$ & $\mathrm{PH}$ & $\mathrm{DO} 2$ & $\mathrm{NH}_{3}$ & $\mathrm{NO}_{2}$ & $\mathrm{NO}_{3}$ & $\mathrm{PO}_{4}$ & $\mathrm{SiO}_{4}$ & $\mathrm{TOC}$ & $\mathrm{Ni}$ & $\mathrm{Mn}$ & $\begin{array}{l}\mathrm{Ni}_{-} \\
\mathrm{Sed}\end{array}$ & $\begin{array}{l}\mathrm{Cd} \\
\text { Sed }\end{array}$ \\
\hline $\begin{array}{l}\text { Spon } \\
\text { ges }\end{array}$ & & & & & & & & & & & & & 0.63 & & \\
\hline Nematoda & & & & & & & & & & & & & 0.54 & -0.56 & \\
\hline $\begin{array}{l}\text { Amph } \\
\text { ipoda }\end{array}$ & & & & -0.78 & & 0.84 & 0.69 & & & 0.62 & & & & & \\
\hline $\begin{array}{l}\text { Aniso } \\
\text { poda }\end{array}$ & & & & & & & & & & & & & 0.52 & & \\
\hline $\begin{array}{l}\text { Deca } \\
\text { poda }\end{array}$ & & & & & & & & & & & & & 0.64 & & \\
\hline $\begin{array}{l}\text { Gastr } \\
\text { opoda }\end{array}$ & & -0.80 & -0.80 & & & & & 0.87 & 0.62 & 0.57 & & & & & \\
\hline Bivalvia & 0.53 & & & & 0.54 & & & & & & & & & & \\
\hline Sipunuli & & & & & & & & & & & & & 0.63 & & \\
\hline $\begin{array}{l}\text { Amphi } \\
\text { oxus }\end{array}$ & & & & & & & & & & & 0.58 & -0.61 & -0.52 & & 0.64 \\
\hline $\begin{array}{l}\text { Abun } \\
\text { dance }\end{array}$ & & & & & & & & & & & & & & -0.55 & \\
\hline $\begin{array}{l}\text { No. } \\
\text { species }\end{array}$ & & 0.54 & & & & & & & & & & & & & \\
\hline
\end{tabular}




\section{DISCUSSION}

During the present work along the western coast of Alexandria (ca $60 \mathrm{~km}$ ) from the western harbour to the Marbilla village, great variations and instability in the substrata of biota were reported. Then interaction within stable "benthos patches " may involve a large proportion of surviving individuals. If surviving benthic mobile individuals are concentrated in stable "benthic patches" (either through active refuge seeking or because survival is higher in those "benthic patches"), then impacts of benthic mobile individuals (either as predators or competitors for space) in those benthic patches may have a large influence on overall population at a large area. Monitoring and assessing environmental quality of coastal waters are greatly needed since the ecosystem and abundant communities are among the most sensitive indicators due to any stress factor. As regards to the terms of biological pollutants and biological pollution, have recently discussed the problem caused by invasions species (Boudoureque and Verlaque 2002). A viewpoint, Elliott (2003), mentioned that chemical pollution and pollutants leading to changes in populations, community, and ecosystem as well as due biological pollutants at all levels of biological organization (from cell to ecosystem). He also added that the sea's ability to degrade the sewagederived organisms via dilution, dispersion and killing potentially pathogenic gut microorganisms caused by long-sea sewage outfalls, as in many coastal developed areas. The present study indicates the stress of effluent of various pollutant sources in particular near the two-drainage areas (El Amom and Nobaria drains) flow directly into seawater. At these high entropic areas, the species diversity and benthic densities obviously remained relatively high, whereas at other sampling sites showing similarity complex results, with no simple relationships between disturbance intensity and pollutant impacts on bottom fauna.

The data analyses using diversity indices and total abundance indicated that sites having distinct eutrophic level were highly diverse and had more individual organisms than other sites. This is due to large number of polychaetes worms and free-living nematodes. The polychaete worms are infauna and feed on detritus and some are epifauna. They could be presented in high load of pollution when food is available. Physical and chemical properties of seawater and sediments greatly varied among sites and over time (NIOF, 2006). There were significant correlation between diversity indices and some physiochemical parameters. At most sites, weak correlations were detected, indicating that the organic matter, $\mathrm{Cu}, \mathrm{Pb}, \mathrm{Mn}, \mathrm{SiO}_{4}$, and salinity are possible factors controlling and structuring the communities. The numerical abundance and biomass varied among sites and over the time, these indicated that the major taxonomic groups of benthos were not similar. However, the environment conditions at the coastal region near the sources of disturbances on marine ecosystem had enough stress on the structure of benthic invertebrates. At site 1 in March, site 10 in May, site 13 in July, site 10 in September, sites 5, 16 in November and site 6 in January, the biomass and numerical abundance were significantly higher than in all period of collections. However, no significant differences among the diversity indices, which indicated that the communities are unstable and possible factors, are total organic matter, heavy metals, and salinities at sampling sites. Correlation between diversity values, organic matter, $\mathrm{Mn}, \mathrm{Cu}, \mathrm{SiO}_{4}$ and salinity, temperature indicating significant correlations and may be factors structuring controlled the benthic communities. The same situation observed at sites 1-10 where the occurrence of pollutants is not sensitive to the presence of oligochaetes, nematodes, polychaetes species. 
Dejonge et al. (1993) cited that increased pelagic production in coastal waters due to nutrient enrichment could benefit macrobenthic filter feeders, in shallow water by increasing the food supply. EIMP (2002) reported that the following opportunistic species are associated in area with enriched sediments having a high level of pollutants namely: the Nematode Enoplus sp. was not influenced by enrichment of $\mathrm{Cd}, \mathrm{Hg}$ and sewage loading; the Polychaeta Prionspio auklandica at sites enriched with organic matter, TP, TN and Cd and the Bivalves Donax spp., Macoma cumana, Mactra corralina, Solena marginatus. At the western coast of Alexandria from Nobaria drain to western harbour (EIMP 2002) the mean number of species was 8 with mean abundance of 502 ind. $/ \mathrm{m}^{2}$, where the abundance of free living nematodes, polychaetes and molluscs respectively yielded 396,68 and 3 ind. $/ \mathrm{m}^{2}$.

The present data analysis indicated that the distribution and abundance of macro benthos varied greatly due to the nature of the bottom and the distance from both the sources of pollution and the human coastal activity as reported by El Komi (1996, 1997), El-Komi, and Beltagy (1997). However, the varying degree of faunal affinity is related to the effect of current, land and sea barrier, temperature in inshore water, the duration and period of spawning and the duration of the planktonic larvae (Abott, 1966). The distribution of animals and their species composition, according to Connell (1961), depend upon the nature of substrata that is affected by the degree of competition for space. On the other hand, the substratum provides to be the "Master Factor" as suggested by Thorson (1960) as it is responsible for the settling of bottom invertebrates' larvae. The presence of bottom invertebrate predators is another factor affecting the composition of bottom fauna. Besides, the effect of prevailing environmental conditions influencing on the timing and duration of planktonic larval stages as reported by El-Komi (1996, 1997).

The levels of species abundance, diversity and productivity are the main variables that assess man's impact on the marine environment and are useful in configuring offshore area, where the biology of constituent species is not known. Further quantitative sampling and statistical analysis demonstrate the biological mapping for the spatial distribution patterns of different marine organisms. However, there was directional trend for benthic populations, which may have maximum impact at intermediate disturbance levels. This estimation for the impact of the environment was based on bottom sediments collections using van Veen grab sampler. So no consideration of "benthic patches" was taken which could increase the real biodiversity depending on the numerical abundance and species composition structure of various benthic populations within patches. The benthic mobile individuals at any case reflect the reliable effect of any disturbance on regional distribution of macobenthic communities at a certain ecosystem area. Thus this supports the possibility that encountered occurrence of some epi- and infauna inhabiting soft bottom show no record for "benthic patches" populations because of the difficulty of using van Veen grab bottom sampler to collect from hard bottom, so reduced that benthic fauna to be collected among the bottom sampling.

The numerical density of benthos per square meter was significantly lower (ranged annually from 959 ind.$/ \mathrm{m}^{2}$ to 8444 ind. $/ \mathrm{m}^{2}$ ), reflecting a general pattern of lower bottom invertebrates densities at the different sampling sites. These differences are mainly due to the structure of substrata, where there were no variations between the quality of sediment to the number and biomass of benthic groups. These results do not clearly support the impact of undesirable environmental conditions that explain the trend for reduced benthic biodiversity as the numerical abundance of benthic communities. 
Furthermore, the experimental studies on the impact of different abiotic factors prevailing in the coastal region are needed besides the results of field surveys to determine the relative tolerant level where benthic densities were either unchanged or reduced in variable discharge of pollutants into seawater. These data can provide evidence of increased or decreased resistance rate of common benthic population in certain area of study in variable concentration of experimental treatments. Thus, the relative tolerant level of the discharged pollutants would remain constant and probably decline in variable treatment concentrations. In fact, a definite increase in discharged pollutants did not indicate significant reduction in benthic densities. On the other hand, this level of resistance to certain pollutant seems too varied greatly among different benthic species as well in different sites.

The sediment structure of the western coastal region under study differed at located areas from western harbor of Alexandria to Marbella resort village. This area has different depths extending from shallow water $(1.5-5.0 \mathrm{~m})$ at sites $1,7,11,14 \&$ 16 , to moderate depth $(5.0-8.0 \mathrm{~m})$ at sites $2,4 \& 8$ and relatively deeper water (10-23 m) at sites $3,5,6,9,10,12,13 \& 15$. The nature of bottom sediments is characterized by having sludge/plant fragments habitat at sites $1,2 \& 7$; fine/coarse sand habitat at sites $3,4,5,6,8,9 \& 10$ and fine/calcareous sand habitat at sites 11, 12, 13, 14, 15 \& 16. These variations in type of pollutants are due to the effluent having been discharged into the coastal area (e.g. ca $2.6 \times 10^{9} \mathrm{~m}^{3}$ annually from El Amom drain). Therefore, the area located close to the outfall of the main drains are characterized by having distinct eutrophication level leading to significant increase in the species richness and mean abundance of benthic communities of the tolerant species distinguishing three communities:-sludge/plant fragment community of high biodiversity -fine/coarse sand community of moderate biodiversity -fine/calcareous sand community of low biodiversity.

The water quality and sediment structure have been changed due to outfall of different pollutants through the two main drains having distinct amount of untreated urban sewage, agriculture drainage and industrial wastes; hydrocarbon from SUMED petroleum transportation and waste product from commercial vessels and fishing boats beside military vessels in El Dekhela and western harbours. The study area shows organic enrichment at sites 1, 3-6, 9, 10, 13, 14; low salinity (less than 8\%o) at sites 2, 7 with high $\mathrm{SiO}_{4}$ and $\mathrm{Pb}$ content (NIOF, 2006). In addition, $\mathrm{Pb}$ content was high at sites $1,2,3,7,8$ while $\mathrm{Cu}$ was high at sites $1,2,3,11$. On the other hand, at sites $12,15,16$ located not closed to the outfall does not show distinct enrichment in the chemical characters.

Sustainability of marine ecosystems that degrades their functioning can be related to: development of periodic and permanent hypoxia that may occur naturally; fishing pressure and eutrophication, dependant upon the hydrodynamics of the area studied. According to Rydberg $(1982,1984)$ in areas with low water exchange, high organic deposition, oxygen content below the halocline and about 1-2 $\mathrm{m}$ above the seabed were below $2 \mathrm{ml} / 1$ with hydrogen sulphide sometimes developing. Gray et al. (2002) suggested that under extreme eutrophic conditions where Oxygen falls to below $0.5 \mathrm{mg} / \mathrm{l}$, sediments often become anoxic with redox potential discontinuity layer rising to near the surface and the consequent release of ammonia and hydrogen sulphide into water column and such changes are catastrophic for benthic and pelagic organisms.

The main disturbances in the study area concerning the coastal development impacts on macrobenthos included seabed disturbance by fisheries gear. According to QSR (2000), the distribution of the macrofaunal communities reflect the nature of 
sediments, the depth of water and the latitude as expected as the influence of external factors, including human activities. Increased input of organic matter (from wood pulp waste and sewage sludge) is one of main consequence of eutrophication for benthos (Pearson and Rosenberg, 1978). According to Nixon, (1995) eutrophication as an increase in the rate of supply of organic matter to an ecosystem, regardless of its source of origin may locally produce or import organic carbon. Macrobenthos communities as a result to eutrophication include changes in community structure and disturbance or death due to anoxic condition. Species numbers, abundance and biomass are usually reduced during hypoxia, but there are some exceptions (Diaz and Rosenberg, 1995, Gray et al., 2002). The values of indicators of benthic changes for making management decisions at various scales (Quintino et al. 2006) are difficult of deriving and using qualitative and quantitative indicators from benthic communities in stable, and in moderately and highly variable environmental conditions in the estuarine, coastal and open sea habitats.

\section{CONCLUSION}

-The variations in the regional and monthly distribution of benthic fauna in the study area revealed that there are markedly variations.

-The area in front to the sources of pollution namely El Amom and Nobaria drains appeared with pronounced increase in the abundance and biomass of benthos population rather than at the western sector due to opportunistic species.

-The data analyses indicated that the macro benthos was directly influenced by highly increasing levels of pollutants on the bottom.

-In general, the present results indicate that the pollution in the different sites has more effect on the occurrence of macro benthos invertebrates.

-Organic matter contents in seawater may be a factor structuring diversity values with increasing total organic matter.

-Possible controlling factors include the structure of sediment and content of various elements of heavy metals, organic hydrocarbons in sediments which directly affect the growth of bottom living organisms.

-The reference sites away from near shore also indicated high variations in physical and chemical environmental parameters, sediments and composition of benthic structure and generally fluctuations in numerical abundance and biomass of benthic communities.

-The previous studies of the area in front of Alexandria to Abu Qir Bay (EIMP 2002) indicated high diversity indices in the benthic communities.

-Continued monitoring of these communities is needed to determine the short and long-term changes in the marine ecosystem essential for fisheries management along the coastal region.

\section{REFERENCES}

Abott D.P. (1966). Factors influencing the zoogeographic affinities of the Galapagosinshore marine fauna. Proceedings of the Symposium on Galapagos International Scientific Projects, 108-122.

Borja, A. and I. Muxika, (2005). Do benthic tools respond to all impact sources? The Case of AMBI (AZTI Marine Biotic Index). In indicators of stress in the marine 
benthos, UNESCO, Intergovernmental Oceanographic Commission Workshop Report No. 195

Boudoureque, C. F. and Verlaque, V. M. (2002). Biological pollution invasions. Ecol. Eco., 39(2): 197-202.

Connell, J.H. (1961). Effects of competition perdition by Thais lapillus and Other factors on nature population of barnacles Balanus balanoides. Ecol. Monog., 31: 61-104.

Dejonge, V. N.; Essink, K. and Boddeke, R. (1993). The Dutch Wadden sea - a changed ecosystem Hydro., 265: 45-71.

Diaz, R. J. and Rosenberg, R. (1995). Marine Benthic Hypoxia. A review of its Ecological Effects and the Behavioral Responses of Benthic Macrofauna. Oceanography and Marine Biology: An Annual Review, 33: 245-303.

EIMP (2002). Draft Report On Sediments, Biota and Benthos Data from Coastal Areas of the Gulf of Suez and Mediterranean Sea during 2000.

El-Komi, M. M. (1996). Coastal development and pollution impacts on the distribution of macrobenthic communities along the eastern coast of the Gulf of Suez (Egypt). P. J. Mar. Sci., 5(1): 1-13.

El-Komi, M. M. (1997). A preliminary list of the summer macrobenthos in the intertidal one of the western Gulf of Suez. Bull. Inst. Oceanogr. and Fish. ARE, 23: 295-314.

El-Komi M. M. and Beltagy A. I. (1997). Distribution of macro benthos assemblages in the north coast of Egypt. Bull. Inst. Oceanogr. and Fish. ARE, 23: 267-293

Elliott, M. (2003). Biological pollutants and Biological pollution-an increasing Cause for concern. Mar. Poll. Bull., 46: 275-280.

Gray, J.S.; Wu, R.S. and Or, Y.Y. (2002). Effects of hypoxia and organic enrichment on the Coastal Marine Environment. Mar. Ecol. Prog. Ser. 238: 249-279.

Laetz, C. (1998). Marine Benthic Invertebrate Communities Near King County's Wastewater Outfalls. Puget Sound Research, 754-759.

Lake, P.S. (2000). Disturbance, patchiness, and diversity in stress. J. North Amer. Bentholog. Soc., 19: 573-592.

NIOF, (2006). Final Report on Effects of Industrial, Touristic Activities and Marine Transport on Physical, Chemical and Biological Characteristics of water and Fish Populations West of Alexandria

Nixon, S.W. (1995). Coastal marine eutrophication a definition, Social Cause, and Future Concerns. Ophelia, 41: 199-219. 
Pearson, T.H. and Rosenberg, R. (1978). A Comparative study of the effects on the Marine Environment of Wastes from Cellulose Industries in Scotland and Sweden. Ambio, 5: 77-79.

Pielou, C. (1966). The Measurement of diversity of different types of Biological Collections. J. Theor. Biol., 13:131-144.

PTI, (1993). Recommendations for Assessing Adverse Benthic Effects in Puget Sound. Perpard for: Washington State Department of Ecology Sediment Management Unit, Olympia, WA.

Quintino, V.; Elliott, M. and Rodrigues, A. M. (2006). The Derivation, Performance and Role of Univariate and Multivariate Indicators of Benthos Change: Case Studies at Different Spatial Scales. J. Exper.1 Mar. Biol. and Ecol., 330: 368-382.

QSR, (2000). Chapter 5 in OSPAR Commission for protection of the Marine Environment of the North-East Atlantic: Quality Status Report 2000, Region III Celtic Seas.

Rees, H.L.; Sneddon, J. and Boyd, S. E. (2005). Benthic Indicators: Criteria for Evaluation Scientific and Management Effectiveness. In Indicators of Stress in the Marine Benthos, UNESCO, Intergovernmental Oceanographic Commission Workshop Report No. 195

Rumohr, H. (2005). A 5-Step Succession Model for the Baltic - A Future Management Tool? In Indicators of Stress in the Marine Benthos, UNESCO, Intergovernmental Oceanographic Commission Workshop Report No. 195

Rydberg, L. (1982). Nutrient conditions and nutrient flow within the southeastern Kattegat. Vatten, 38: 436-450.

Rydberg, L. (1984). Some observations of nutrient fluxes through the coastal zone. ICES, C.M. 1984/No.62, 20 pp.

Shannon, C. E. and Weaver, W. (1949). The Mathematical Theory of Communities. University of Illinois Press: 117 pp.

Shine, J. (2005). A Summary of Results if IOC-BIG Benthic-TOC Study. In Indicators of Stress in the Marine Benthos, UNESCO, Intergovernmental Oceanographic Commission Workshop Report No. 195

Thomson, J. R.; Lake, P. S. and Downes, B. J. (2002). The Effect of Hydrological Disturbance on the Impact of a Benthic Invertebrate Predator. Ecology, 83(3): 628-642.

Thorson, N.G. (1960). Parallel level-bottom communities their temperature adaptation and their "balance" between predators and food animals. In perspective in Marine Biology ed. A.A. Buzzuti-Traverso, 76-86. 


\section{ARABIC SUMMARY}

\section{توزيع مجتمعات الأحياء القاعية المتأثرة بالنشاط البشرى بالساحل الغربي للإسكندرية ـالبحر المتوسط ـمصر محمد محمد الكومى البحم \\ المعهد القومى لعلوم البحار و المصايد ، الإسكندرية ، مصدر ، مصر \\ Komimohamed50@yahoo.come}

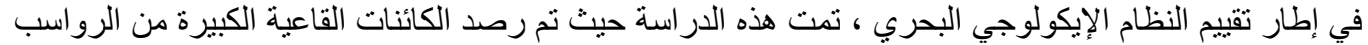

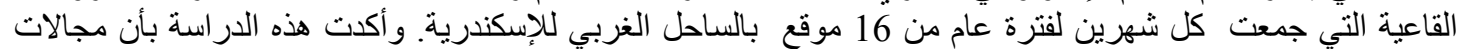

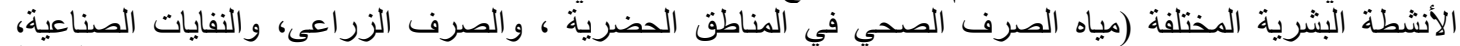

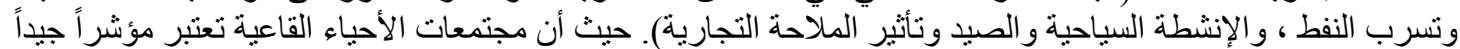

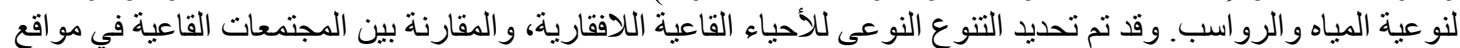

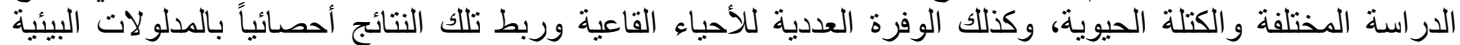

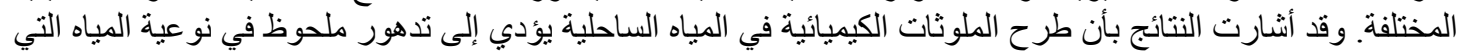

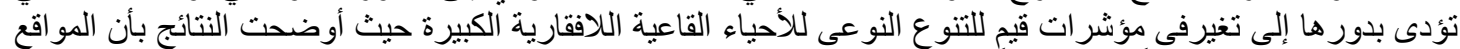

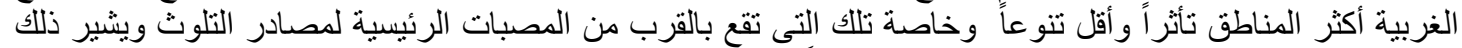

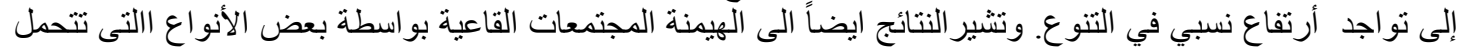

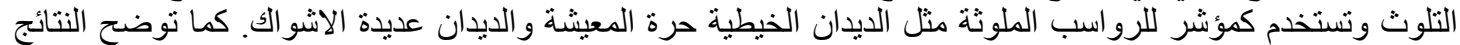

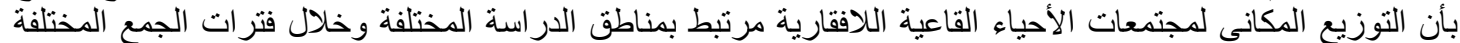

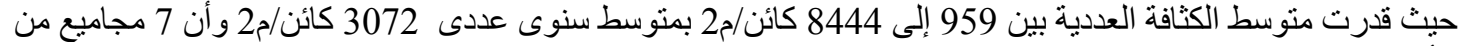
الأحياء القاعية يمكن وضعها فئ تسلسل تتابعى كالتالى :

Nematodes $46 \%>$ polychaetes $22 \%>$ amphipods $13.8 \%>$ cirripedes $4.8 \%>$ oligochaetes $4 \%>$ gastropods $3.0 \%>$ bivalves $1.5 \%$.

من ناحية أخرى فإن منوسط كثافة الكتلة الحية من مجتمعات الأحياء القاعية اللافقارية قدرت بـ 155 جم/م2 وان 6 من من 6 من

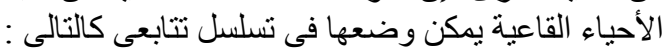

Cirripedes 33.5\% > Algae 30.6\% > Gastropods 12.7\% > Bivalves 9.1\% > Sea grass 7.0\% > Polychaetes $1.7 \%$.

وفي مواقع الدراسة المختلفة يتضح بأن الهيكل العام لعدد الأنواع من المجتماعات القاعية توضح بأن الديدان

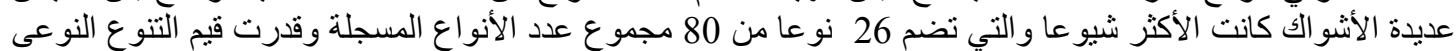

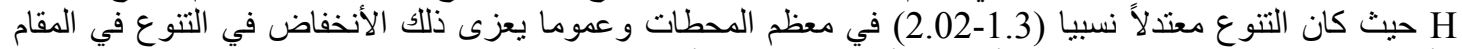

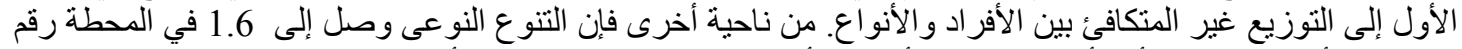

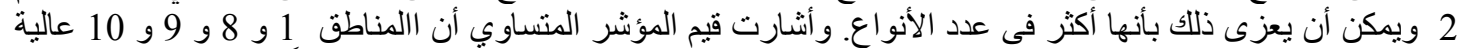

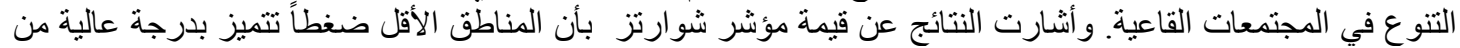

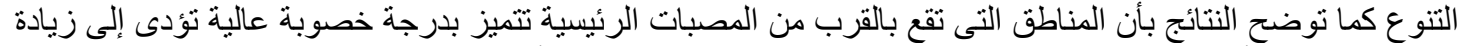

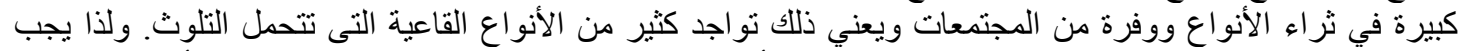

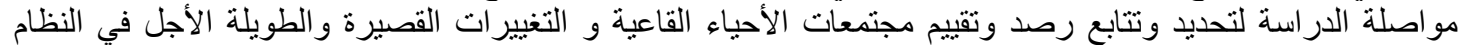
البيئي البحري الذي يعتبر أساسيا لإدارة مصائد الأسماك على طول ولفئ المنطقة الساحلية. 